\title{
Improvement of a Joystick Controller for Electric Wheelchair User
}

\author{
Masatomo Shibata1, Chao Zhang1, Takakazu Ishimatsu', Motohiro Tanaka², Joel Palomino³ \\ ${ }^{1}$ Nagasaki University Graduate School of Engineering, Nagasaki University, Nagasaki, Japan \\ ${ }^{2}$ Department of Transportation Mechanics, Kurume Institute of Technology, Kurume, Japan \\ ${ }^{3}$ Pontificia Universidad Catolica del Peru, Lima, Peru \\ Email:m-shibata@zeshinkai.or.jp, bb52311103@cc.nagasaki-u.ac.jp,ishi@nagasaki-u.ac.jp, \\ tanamo@kurume-it.ac.jp, j.palomino@pucp.edu.pe
}

Received 24 September 2015; accepted 21 November 2015; published 25 November 2015

Copyright (C) 2015 by authors and Scientific Research Publishing Inc.

This work is licensed under the Creative Commons Attribution International License (CC BY).

http://creativecommons.org/licenses/by/4.0/

(c) (i) Open Access

\begin{abstract}
The electric wheelchair is an effective machine for people with lower limb disability. The user controls the wheelchair by a joystick that helps the user to navigate the wheelchair along the desired path. Suppose the user on the wheelchair wants to operate the computer for his jobs or enjoyment, it is preferable for the user to be able to operate the computer without transferring from the wheelchair to a computer desk. Of course, some computer input devices are available for wheelchairs. One reasonable idea is to use the familiar joystick on the wheelchair as a computer input device. In this paper a joystick controller is proposed, which enables the user on the wheelchair to operate the computer settled on a nearby table. The proposed joystick controller can be achieved by mounting the sensor unit on the joystick without any modification of the conventional wheelchair controller. The principle of the sensing unit is to measure the inclination angle and the direction of the joystick with an acceleration and gyro sensor. Then the sensing unit sends the control data to the computer via an infrared or wireless signal. This proposal is based on a request done by the wheelchair users.
\end{abstract}

\section{Keywords}

Wheelchair, Joystick, Communication, Computer Interface

\section{Introduction}

For the people with lower limb disability many electric wheelchairs are commercialized and some of them use a joystick controller. These wheelchairs make their daily live independent and active. In these wheelchairs the us- 
ers who suffer upper limb disability often manipulate the joystick with their chin. On the other hand, other wheelchairs use the body vital signals to operate the wheelchair. In a research of Fukuda et al. [1], a device to operate the computer using the patients' slight body movements was developed, this device used the head movements and eyebrow movements to operate the computer in ALS patients. Also in the research of Takami et al. [2], a device that uses image processing is developed to operate the computer, a feature of this device is that it's not necessary to mount a device on the patients head. Using the same working principles the wheelchair can be operated with the user's vital signals. However, the joystick lever is still admitted as practical and user friendly tool. It is important to notice that the manipulation of the joystick can be recognized as a good physical training and also as a mean to live an independent life. In Figure 1 an electric wheelchair user who suffers cerebral palsy is shown. Even though his four limbs are paralyzed he can operate the joystick with his chin and enjoy an active life with the help of the wheelchair. In order to further improve their quality of lives, assistive devices need to be developed to enable communication. Such development is becoming easier and cheaper since the surrounding technologies are developing.

In this paper a joystick controller for wheelchair users is proposed, the developed device enables the wheelchair user to operate the computer on nearby desk. A distinguishable feature is that the operation of the computer can be achieved by using the same joystick mounted on the wheelchair without modifying the conventional electric wheelchair. This is achieved by mounting the developed sensing unit on the joystick. After this the device sends the sensed signal to a mouse controller device located on the computer.

As everyone knows, the computer is a convenient and useful device for people with physical disabilities. They often use computers as a communication device and also as an environmental control device for home appliances such as TV, air conditioner and so on. People with physical disabilities often desire to use the computer under various conditions. The wheelchair user in Figure 1 who suffered cerebral palsy expected to operate the computer without transferring to a computer desk. Every time he wanted to operate the computer, he must ask the care-giver to mount another joy stick on the wheelchair. His desire was to be independent from care-givers.

Additionally another wheelchair user with slight upper limb disability, who was engaged in computer design, also requested a joystick to operate the computer. He tried to operate the graphic design software with various joysticks without satisfactory results. Proper settlement of the joystick and also training with the new joystick are important. After several trials it became clear that the joystick on his wheelchair was an appropriate computer interface for his designing task since he was already accustomed with this joystick in his daily life on the wheelchair.

For the wheelchair users that employ a joystick lever, the joystick is also expected to be used as an interface for the computer. By mounting a sensing unit on the joystick controller on the wheelchair, the computer operation can be achieved. In this paper the performance of the developed device is compared with other devices that use vital signals to operate the computer.

\section{Computer Interface to Use Joystick on the Wheelchair}

\subsection{System Configuration of Joystick Controller}

In order to enable the joystick controller on the wheelchair to work as a computer interface, a new sensing unit mounted on the joystick controller is proposed as shown in Figure 2. The joystick sensing unit obtains the data

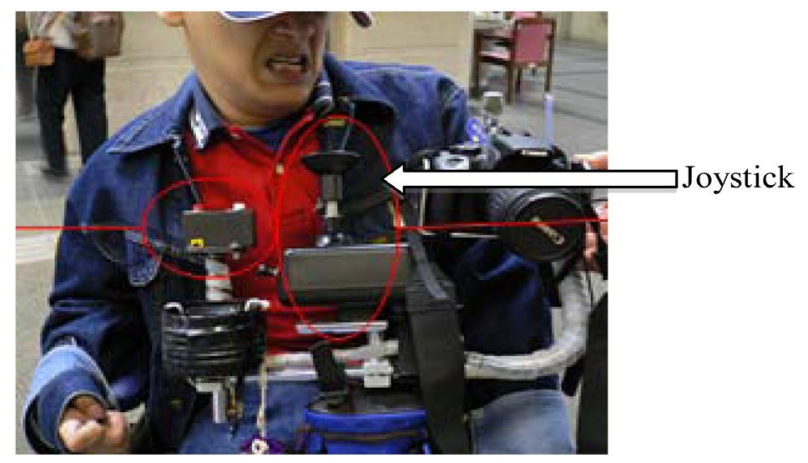

Figure 1. Wheelchair user using a chin control device. 


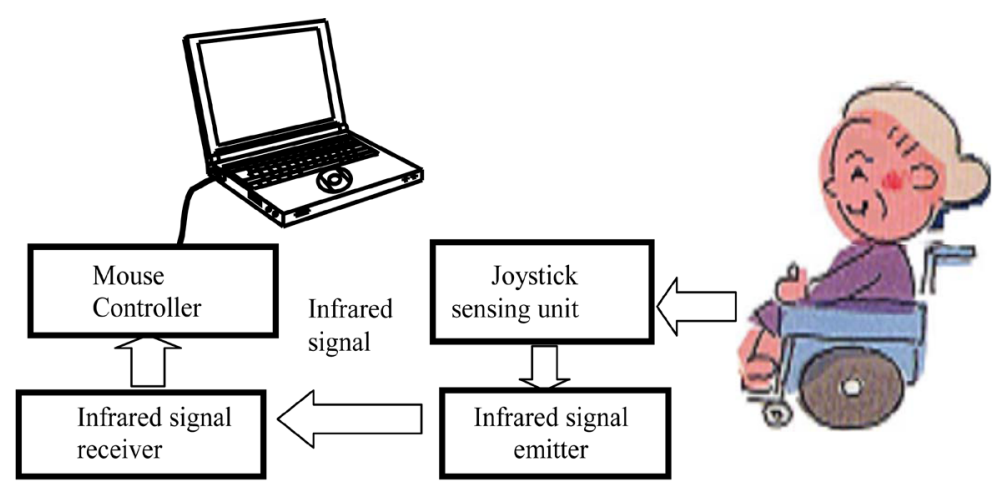

Figure 2. System configuration.

related to the joystick like inclination angle and orientation.

The sensing unit is composed of an acceleration sensor, gyro sensor, microprocessorPIC16F88 and one Infrared port. The acceleration and gyro sensor measures the joystick angle movement and direction, this signal is goes into the microprocessor analog to digital converter which converts the electric input signal to angle and direction. After this the microprocessor sends this data to the mouse controller through the infrared port.

The mouse controller consists of a microprocessor PIC18F2550 and one Infrared port. The processor receives the joystick information through the infrared port and converts the information into mouse commands through an HID Mouse library. This library from Microchip [3] enables the microprocessor to generate the same signals of a computer mouse. So there is no need to install additional software unto the computer, just to plug the device to the computer USB port. Finally three buttons near the joystick are used to activate the click actions of the computer mouse.

The joystick sensing unit is shown in Figure 3. The inclination angle of the joystick can be obtained based on the principle of the detected data by the acceleration sensor. By introducing three-dimensional acceleration sensor, inclination angle and direction of the gravity can be obtained. It is important to note that the inclination angle and direction obtained are affected by the acceleration of the sensor. By incorporating the gyro-sensor, the inclination angle and direction of the joystick can be estimated by suppressing the effect of the acceleration.

The equation to estimate inclination angle $\theta_{k+1}$ at $k$ sampling time is as follows [4]:

$$
\theta_{k+1}=\theta_{k}-\omega \theta_{k} \Delta t+\omega \theta_{a c c} \Delta t+\theta_{\text {gyro }} \Delta t,
$$

where $\omega$ is cutoff frequency, $\Delta t$ is sampling interval, $\theta_{\text {acc }}$ is data obtained by acceleration sensor, $\theta_{\text {gyro }}$ is data obtained by gyro sensor.

\subsection{Experiments of the Sensing Unit}

Experiments to evaluate the applicability of sensing unit were conducted as shown in Figure 4.

In the first experiment the effect of introducing the gyro-sensor is discussed. The acceleration data gather during the joystick operation by the user shown in Figure 1 was obtained as shown in Figure 5 . The dotted line shows the data obtained only by the acceleration sensor. The solid line shows estimated inclination angle compensated by Equation (1). The acceleration data reveal sudden standing up and falling down phenomena of the joystick. By incorporating the gyro-sensor data, the inclination curve was modified to a smooth curve.

In the second experiment the joystick was inclined to the maximum angle (18 degree) toward the right and rotated to make one circle. The data estimated is shown in Figure 6. In this figure the inclination angle could be estimated with accuracy. Considering that some conventional wheelchairs can be operated with the on/off signal of the joystick, accuracy of this sensing unit is enough to navigate the wheelchair with the inclination angle and orientation data from the joystick.

In the third experiment the user was requested to move the computer cursor along the square trajectory $(600 \times$ 512 pixels) on the computer monitor. The results of this experiment are shown in Figure 7, where the trajectory of the cursor is shown as a solid line. And the dotted line shows the target square trajectory.

The trajectory of the cursor shows some deviation from the target path in the right down part, but with a proper joystick operation by the user the trajectory returned to the target square trajectory. 


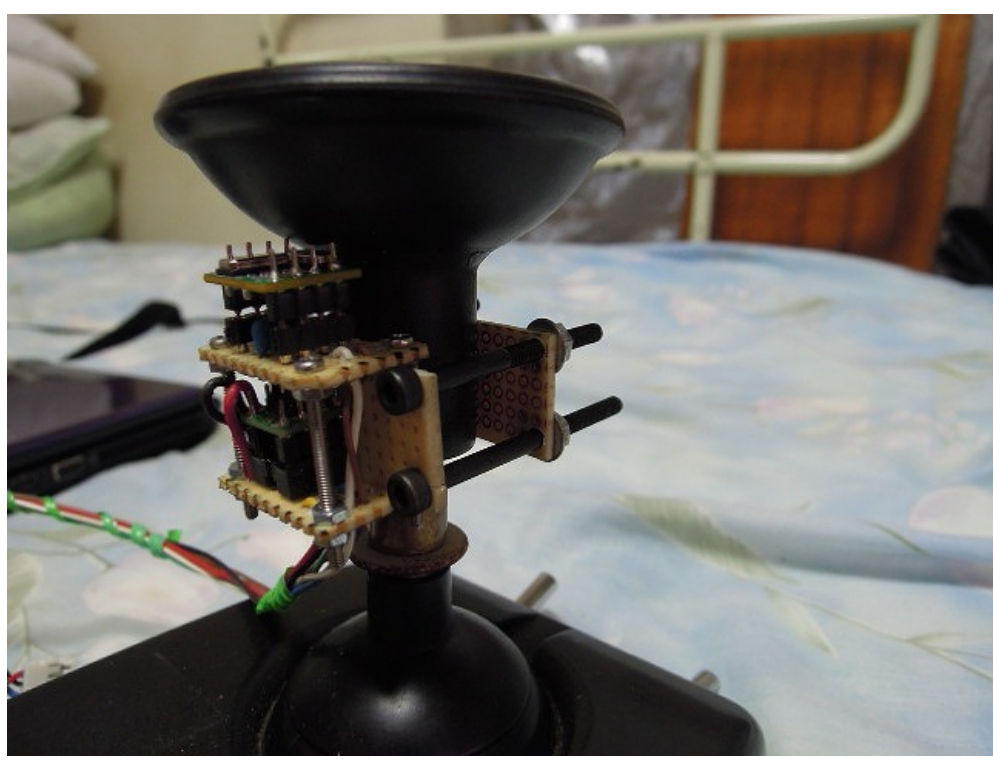

Figure 3. Joystick with acceleration and gyro-sensor.

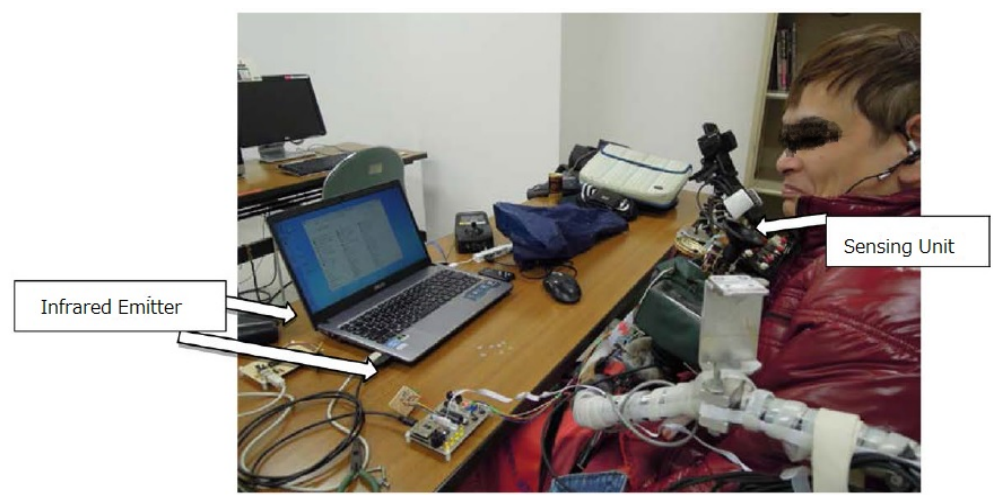

Figure 4. Experiment using proposed joystick controller.

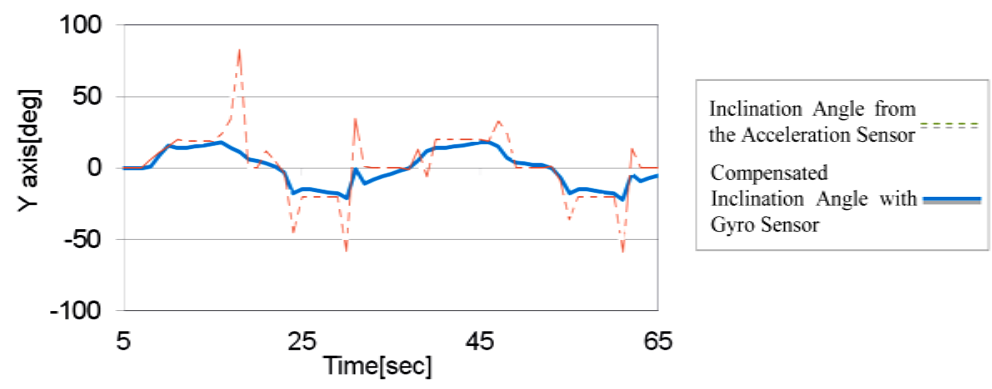

Figure 5. Estimated inclination angle and acceleration data.

Considering these experimental data, the mouse control speed and the direction are settled in proportion to the joystick movement. It should be noticed that the joystick is used as a computer interface while the wheelchair is not running.

The fourth experiment consisted of typing task to write a message by selecting alphabets letters on a screen keyboard three students without any physical disability and the user introduced in Figure 1 performed this experiment by using their chin movement.

The message in Japanese was "kon-nnichiwakyoumogenkidesu” meaning "Good morning. I am fine today". 
All the examinees succeeded in this task in less than forty seconds as shown in Table 1. It is interesting to note that the wheelchair user who suffers cerebral palsy could finish this task with a short delay time respect to the others.

The performance of proposed joystick controller was compared with other computer input devices. The first device [5] uses image processing to detect the users lips and moves the mouse cursor based on the mouth movement, the second [6] one detects the head movement on a pillow to move the cursor according to heads movement and the last one [7] uses the patients head movement through a sensing unit mounted on the head to operate the computer based on the head movements. The typing time of the developed device was compared with the time from the previous described sensors.

The average time to select one alphabet letter was obtained from the typing experiment; the results are shown in Table 2. The experimental conditions were the same. Average time to select one alphabet was between 3.4 second and 5.4 second. The comparison means that these devices have not remarkable differences and that the proposed joystick controller is applicable as a computer input device.

\subsection{Mechanical Sensing Unit of Joystick}

Sensing unit can be achieved by using mechanical switches. In Figure 8 a mechanical sensing unit of the joystick

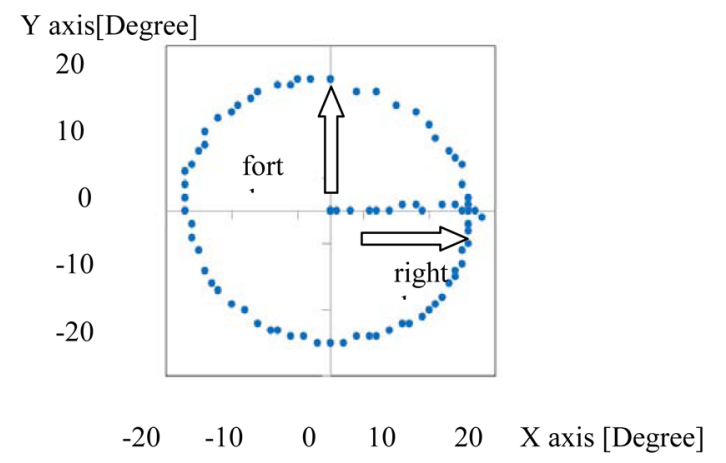

Figure 6. Inclination data of joystick.

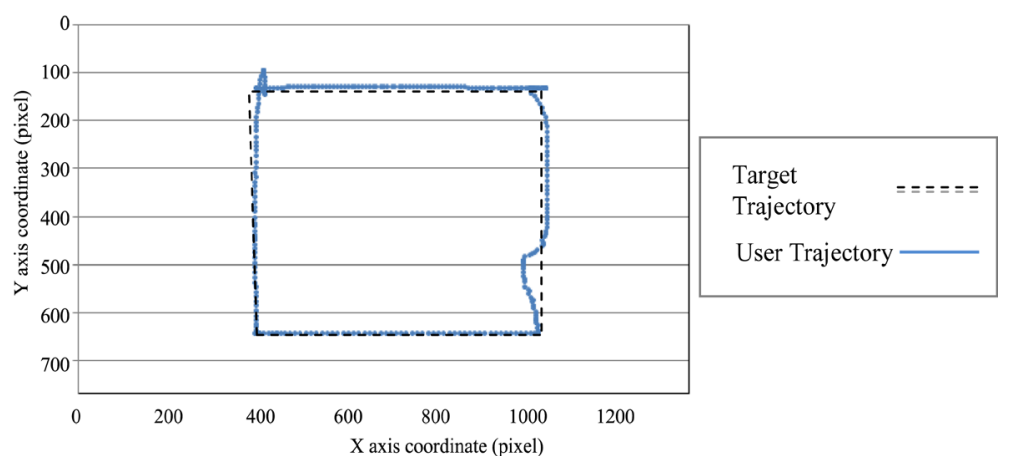

Figure 7. Tracing test using the proposed joystick controller.

Table 1. Required time for typing message.

\begin{tabular}{ccccc}
\hline Examinee & A & B & C & D \\
Required time & $38.8 \mathrm{sec}$ & $34.8 \mathrm{sec}$ & $30.2 \mathrm{sec}$ & $40.2 \mathrm{sec}$ \\
\hline
\end{tabular}

Table 2. Average time to select one alphabet.

\begin{tabular}{ccccc}
\hline Input Device & Proposed Joystick & Image Processing [5] & Pillow Type [6] & Face Mount Type [7] \\
\hline Average time & $4.5 \mathrm{sec}$ & $3.9 \mathrm{sec}$ & $5.4 \mathrm{sec}$ & $3.4 \mathrm{sec}$ \\
\hline
\end{tabular}




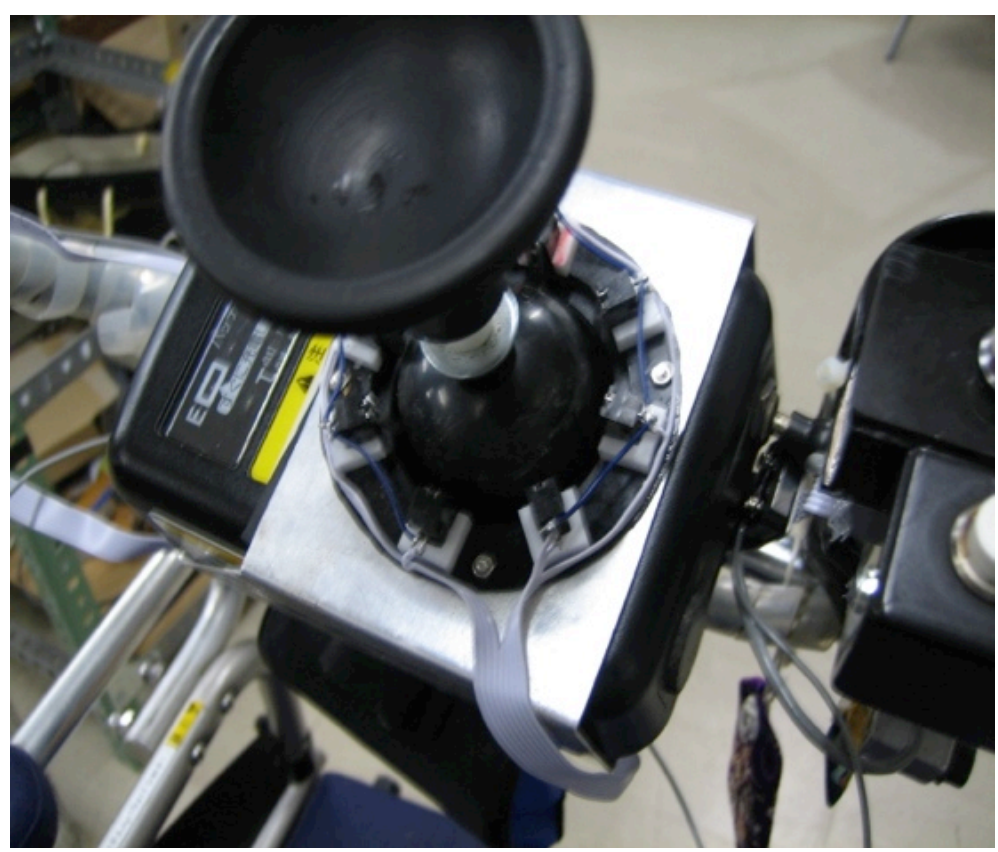

Figure 8. Joystick with eight limit-switches.

is shown. Eight limit-switches are arranged around the joystick. Based on the on/off signals of the eight limit switches the inclination and the orientation of the joystick can be detected. As everyone notices, the resolution of this sensing unit is limited. But from feedbacks of the wheelchair user, he could use this device to operate the computer and also navigate the wheelchair.

\section{Conclusions}

In this paper, a joystick controller system to operate the computer was developed. One distinguishable feature of the system is that the system is mounted on the wheelchair joystick not requiring additional equipment. It is important to note since the joystick of the wheelchair is familiar to the user, the joystick of the wheelchair can be a friendly and a proper computer interface for the user with physical disability.

The functions of the joystick system were evaluated by tracking tests and also by a typing test. The proposed joystick controller has enough accuracy to write texts and select menu items on the computer screen. In this paper while the joystick controller employed infrared communication, Blue Tooth communication could also be employed.

The user shown in Figure 1 has used the proposed joystick system for more than three years. He is satisfied with the functions of the joystick system. He enjoys navigating the wheelchair and operating the computer to write messages and edit pictures.

\section{References}

[1] Fukuda, Y., Tanaka, M., Moromugi, S. and Ishimatsu T. (2003) Communication Device or Disabled Slight Movement Capabilities. International Journal of Human-friendly Welfare Robotic Systems, 4, 7-12.

[2] Takami, O., Morimoto, K., Ochiai, T. and Ishimatsu T. (1995) Computer Interface to Use Head and Eyeball Movement for Handicapped People. IEEE International Conference of System, Man and Cybernetics, Vancouver, 22-25 October 1995, Vol. 2, 1119-1123. http://dx.doi.org/10.1109/icsmc.1995.537920

[3] Microchip Technology Inc. (2014) MPLAB Harmony Integrated Software Framework. http://www.microchip.com/pagehandler/en us/devtools/mplabharmony/home.html

[4] Bernstein, J. (2003) An Overview of MEMS Inertial Sensing Technology. Corning-IntelliSense Corp. http://www.sensorsmag.com/sensors/acceleration-vibration/an-overview-mems-inertial-sensing-technology-970

[5] Zhang, C., Ishimatsu, T., Yu, J.L., Lawn, M. and Shi, L. (2015) Vision-based Displacement Sensor for People with Serious Spinal Cord Injury. 2015 IEEE International Conference on Mechatronics and Automation (ICMA), Beijing, 2-5 
August 2015, 772-777.

[6] Yu, J., Ishimatsu, T., Murray, L. and Tanaka, M. (2015) Pillow-Type Computer Input Device for Serious Spinal Cord Injury. Modern Mechanical Engineering, 5, 61-68. http://dx.doi.org/10.4236/mme.2015.53006

[7] Yu, J., Ishimatsu, T., Shiraishi, N., Lawn, M., Zhang, C. and Tanaka, M. (2015) Face Mount Computer Input Device for Serious Disabled. 2015 IEEE 5th International Conference on Big data and Cloud Computing (BDCloud), Dalian, 26-28 August 2015, 195-198. http://dx.doi.org/10.1109/BDCloud.2015.29 